PAPER

\title{
Analysis of Relevant Quality Metrics and Physical Parameters in Softness Perception and Assessment System
}

\author{
Zhiyu SHAO $^{\dagger \mathrm{a})}$, Member, Juan $\mathrm{WU}^{\dagger \mathrm{b})}$, and Qiangqiang OUYANG ${ }^{\dagger \mathrm{c})}$, Nonmembers
}

\begin{abstract}
SUMMARY Many quality metrics have been proposed for the compliance perception to assess haptic device performance and perceived results. Perceived compliance may be influenced by factors such as object properties, experimental conditions and human perceptual habits. In this paper, analysis of softness perception was conducted to find out relevant quality metrics dominating in the compliance perception system and their correlation with perception results, by expressing these metrics by basic physical parameters that characterizing these factors. Based on three psychophysical experiments, just noticeable differences (JNDs) for perceived softness of combination of different stiffness coefficients and damping levels rendered by haptic devices were analyzed. Interaction data during the interaction process were recorded and analyzed. Preliminary experimental results show that the discrimination ability of softness perception changes with the ratio of damping to stiffness when subjects exploring at their habitual speed. Analysis results indicate that quality metrics of Rate-hardness, Extended Rate-hardness and ratio of damping to stiffness have high correlation for perceived results. Further analysis results show that parameters that reflecting object properties (stiffness, damping), experimental conditions (force bandwidth) and human perceptual habits (initial speed, maximum force change rate) lead to the change of these quality metrics, which then bring different perceptual feeling and finally result in the change of discrimination ability. Findings in this paper may provide a better understanding of softness perception and useful guidance in improvement of haptic and teleoperation devices.

key words: softness perception, quality metrics, haptic rendering device, human-computer interaction, perception model, physical parameters
\end{abstract}

\section{Introduction}

Compliance is an important haptic feeling in our daily life. "Hardness" and "softness" are words to describe subjective perception that people have when they handle hard or soft materials [1]. Stiffness and Youngs modulus are the most popular parameters to measure hardness and softness [2], and both of them can be expressed by metrics of force and deformation, which are generally regarded as two basic physical parameters in the perception of hardness or softness. However, the subjective hardness and softness feelings are not exactly equal to the stiffness of the materials. Perception results are often influenced by experimental conditions, interactive methods and other attributes of the object such

Manuscript received October 22, 2018.

Manuscript revised April 1, 2019.

Manuscript publicized June 11, 2019.

${ }^{\dagger}$ The authors are with the State Key Laboratory for Bioelectronics, Robotic Sensor and Control Laboratory, School of Instrument Science and Engineering, Southeast University, No.2, Sipailou, Nanjing, China.

a) E-mail: 230149550@ seu.edu.cn

b) E-mail: juanwuseu@ @eu.edu.cn (Corresponding author)

c) E-mail: 230169559@ seu.edu.cn

DOI: $10.1587 /$ transinf.2018EDP7358 as damping beside stiffness and Youngs modulus. Damping is ubiquitous in the softness perception in our daily life. Mass-spring-damper is a basic system visible everywhere in design and implementation of mechanical devices. And damping is also widely used in the compliance rendering to achieve better rendering effect. In many situations, users perceive the compliance rendering by exploring virtual environment through haptic devices. To overcome the instability and limitation of the achievable stiffness in teleoperation system caused by communication delays, a common way is to add damping into the system through the controller [3]-[5]. Nuño et al. [6] have also shown that one effective method to guarantee the stability is to guarantee the ratio of adding damping by the controller and the proportional controller gain is bounded from below.

What are relevant quality metrics dominating in the softness perception? How do they reflect the changes of experimental conditions and assessment results? This paper is aiming at simulating interactive effect of stiffness and damping at different levels by haptic rendering devices to learn relevant physical metrics for the softness perception and their working mechanism during the interaction process based on psychophysical experiments and data analysis.

Research on human subjective perception of compliance has a long history. In psychophysical experiments, perceived magnitude and JNDs are important metrics to measure perception ability of human or the performance of rendering. For stiffness, Harper and Stevens [7] performed a magnitude estimation experiment by letting subjects squeeze different types of rubber and reported that stiffness and perceived hardness are related through a power function with an exponent of 0.8. Discrimination experiments were pioneered by Coppen and Scott Blair, they reported that the discrimination ability for stiffness was about $13 \%$, which could be interpreted as the Weber Fraction. Following researchers tried to measure the JNDs for stiffness of different conditions. The average JNDs for compliance that Tan [8] obtained from experiments in which both work and terminal force cues as well as compliance cues were available to the subject was $8 \%$. And they increased to $15 \%$ to 99\% when force and/or work cues were reduced or eliminated [9]. Koçak et al. analyzed the JNDs of compliance in three modes of comparison [10] and showed a significantly better discrimination performance in the case of continuous pressure (discriminate a stiffness change during exploration without taking the probe away from the object).

At the same time, factors affecting the hardness and 
softness perception were also studied. Freyberger and Färber [11] found that discrimination ability would be a little better when samples were pressed down on with one finger compared to when they were pinched between the thumb and index finger. For the hardness and softness perception, both cutaneous and kinaesthetic information is necessary, which had been confirmed by Srinivasan and LaMotte [12]. Further research conducted by Bergmann Tiest and Kappers [13] quantitatively analyzed the contribution percent of kinaesthetic and cutaneous information to the hardness discrimination. They showed that about $90 \%$ of the information comes from surface deformation cues, and $10 \%$ from force/displacement cues. Researches have verified that perceived results of compliance are influenced by different parameters and conditions. Van and Heck [14] investigated the effect of damping on the perception of hardness in two conditions (in-contact task and contact-transition task). Results showed that when injecting damping globally, perceived hardness slightly increased for an in-contact task, while it decreased considerably for a contact-transition task. However, they just paid attention to damping effect on perceived hardness intensity and drew the equal hardness lines, but ignored damping effect on perceptual JNDs and quantitative relations between physical parameters and perceived results.

Many researches were also conducted to find out new useful metrics dominating in hardness and softness perception besides traditional stiffness and Young's modulus. Bergmann Tiest and Kappers [1] showed that subjective perception is based more on stiffness information for the softer stimuli and more on Youngs modulus for the harder stimuli. In the compliance rendering system, stiffness is a physical metric that can be calculated as the force change divided by corresponding deformation and has been proved to be useful for the hardness and softness discrimination. Measured stiffness is usually the average of the stiffness calculated from repeat interaction. And it is mediated by a combination of kinaesthetic (force and displacement) and cutaneous (force) information [2]. Different with Youngs modulus, stiffness is thus not a material property, but a property of the specific object and the way it is being touched [1]. So in the process of actual perception, perceived results may be influenced by other physical metrics that reflecting perception environment, perception methods or contact states beside ratio of force to displacement. Bergmann Tiest [2] also suggested that it would be better to include the more information in haptic display for better rendering. Lawrence and Pao [15] found that the concept of Rate-hardness defined as initial force rate of change divided by initial displacement velocity could reflect the human hardness and softness perception better than stiffness only. Based on which, they achieved high rate-hardness by lead-type compensation in the control system relating measured hand position to commanded force. And they also proposed that this compensation can be interpreted as band-limited damping added to the stiffness rendering. In 2010, Han and Choi [16] proposed the extended rate-hardness which suggested to replace initial force rate of change with maximum force rate of change. They also listed probable useful related metrics in perception of hardness such as Peak Force, Peak Force Rate, Measured Velocity, Measured Stiffness, Original Rate-Hardness, and Extended Rate-Hardness. A correlation matrix was made to show the correlation between physical variables and perceived softness. Hauser and Gerling [17] conducted a series of experiments and confirmed that time-dependent cues playing important roles in the compliance perception. Results showed that the availability of force-rate cues improve compliance discriminability so as to require less deformation of stimulus and finger pad. Higashi et al. [18] analyzed the short-time reaction force caused by tapping various types of objects to investigate physical indicators for estimating subjective hardness. They found that subjective hardness can be predicted by combining the peak force value and impulse of the reaction force. Especially, stiffness and damping factors are associated with the peak force value and impulse of the reaction force respectively. These researches indicated that perceptual compliance may be influenced by many metrics especially time-dependent cues during interaction in most conditions and gave much inspiration on analysis in this paper.

In this paper, the effect of combination of different stiffness coefficient and damping levels on the discrimination ability of softness was investigated by three psychophysical experiments firstly, seen in Sect. 2. Interaction data were recorded during the experiments. Correlation analysis between quality metrics and JNDs was made to find out effective metrics contributing in softness perception, seen in part 3.1, 3.2 and 3.3 of Sect. 3. Then these metrics are expressed by basic physical parameters to analysis their working mechanism. A non-linear regression model is built to try to describe the relationship between physical parameters and perceived results quantitatively, seen in part 3.4 of Sect. 3. In this way, some useful guidance was hoped to be made for haptic data perceptual filtering preprocessing and design of haptic rendering devices for compliance.

\section{Psychophysical Experiments and Results}

Three psychophysical experiments were performed in this paper. In experiment 1 , the effect of exploring speed on the softness perception JNDs was studied. And in experiment 2, the softness rendering perception thresholds were measured at four stiffness levels. In experiment 3 , softness perception thresholds for combination of different stiffness and damping levels were measured.

\subsection{Psychophysical Experiments}

Participants: Twelve subjects (8 males, 4 females) recruited from Southeast University have taken part in the experiments. They were $27 \pm 3$ years old and consented to participate in the experiments. All the participants were right-handed and reported have no loss of haptic sensation or had a history of neurological disorders. They were ig- 


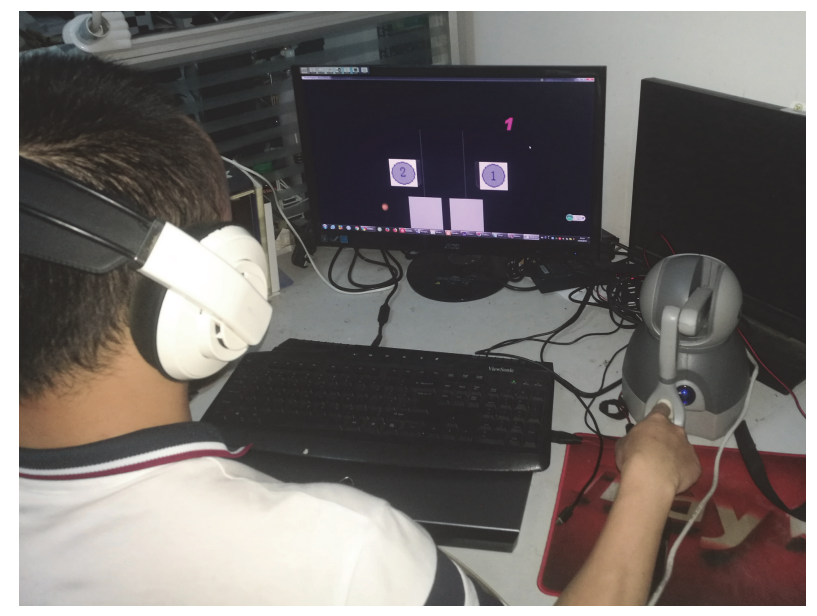

Fig. 1 Interaction with the virtual environment. Participant is sitting in front of the screen and exploring softness rendering in virtual environment, with headphone playing white noise on head.

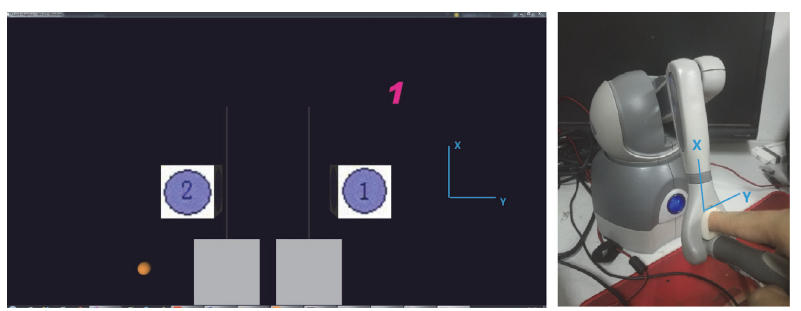

Fig. 2 Haptic interface used in the experiments. Left part is the virtual environment interface, softness effect is rendered in gray blocks and vertical lines are displayed to guide participants exploring along the $\mathrm{X}$-axis as far as possible. Coordinates corresponding to virtual environment are shown in the right part, pressure sensors are placed in the white finger sleeve to record force changing when participant moving the probe up and down along $\mathrm{X}$-axis.

norant about the experiment purpose and compensated for taking part in the experiments. All of them received written instructions and signed an informed-consent form before the experiments.

Stimuli: A Geomagic Touch was used for generating softness feedback in the experiments. The max force can be generated by Touch was $3.3 \mathrm{~N}$ in a 3D space of $160 \mathrm{~mm} \times 120 \mathrm{~mm} \times 70 \mathrm{~mm}$, and the max stiffness for $\mathrm{X}$-axis was $1.12 \mathrm{~N} / \mathrm{mm}$ with the resolution of $0.055 \mathrm{~mm}$, only the force of vertical direction (X-axis) was rendered, perceived and recorded. As shown in Fig. 1 and Fig. 2, the blue ball in the screen was the proxy of the device in virtual environment. When participants pressing the finger sleeve placed on the arms of Touch along X-axis, the blue ball will move in the screen correspondingly. And when the ball moves into the grey blocks where the softness was rendering, the device will give corresponding force feedback. The left brown ball will move up and down at a constant speed in the experiment 2 and 3 to guide the participants exploring at an approximately hoped speed. According to former research experience and conclusion, there are four stiffness levels $(0.025 \mathrm{~N} / \mathrm{mm}, 0.05 \mathrm{~N} / \mathrm{mm}, 0.075 \mathrm{~N} / \mathrm{mm}, 0.1 \mathrm{~N} / \mathrm{mm})$ and four damping levels $(0.0025 \mathrm{Ns} / \mathrm{mm}, 0.005 \mathrm{Ns} / \mathrm{mm}$, $0.0075 \mathrm{Ns} / \mathrm{mm}, 0.01 \mathrm{Ns} / \mathrm{mm}$,) in total in the experiments. In the softness rendering process in this paper, feedback force was generated by stiffness and damping effect together, as shown in Formula 1:

$$
f(t)=f_{s}(t)+f_{d}(t)=K \delta x(t)+B \dot{\delta} x(t)
$$

Here the stiffness force $f_{s}(t)$ is in proportion to the deformation $\delta x(t), K$ is the stiffness and damping force $f_{d}(t)$ is in proportion to the exploring speed $\dot{\delta} x(t), B$ is the damping coefficient. In the pressing stage, stiffness force and damping force are superimposed because they have the same direction while they are mutually inhibiting for the opposite direction in release stage.

Procedure: As shown in Fig. 1, participants seated in front of the experimental desk, while wearing headphones playing white noise to mask the sound of the device in the experiments. When participants were moving the blue ball along $\mathrm{X}$-axis into the grey blocks, the Touch will generate feedback force according to rendering algorithms and make the blocks feel like flexible objects. The grey blocks will not have visual deformation because it was reported that visual deformation would have some effect on haptic perception [19].

The method of constant stimuli was used in the experiments to obtain reliable JNDs. For each reference stimulus which is combined of one stiffness level and one damping level (16 in total), test stimuli are the same damping level with reference combined with 8 stiffness levels evenly ranged from $50 \%$ to $150 \%$ of reference stiffness. Participants were presented with two stimuli in the blocks respectively at the same time for each trail, and the appearance of reference and test stimuli on the left or right side is random. Participants were allowed to explore and switch between blocks of each side as they want when the experiment started. They were asked to move the finger sleeve up and down along the $\mathrm{X}$-axis when exploring inside the blocks because the force feedback were generated only along this direction. Once finished the comparison within a time limit of 30 s, they were forced to make a choice by moving the ball into the button 1 or 2 and click the corresponding button. Button 1 choice meant the right stimulus was harder and button 2 choice meant the left stimulus was harder. After the choice was completed, another pair of softness would appear for the participants to compare. Each compare of test and reference was tested 10 times per subject, thus there are 80 times comparisons in total for each participant per reference stimulus level. Force, position and velocity information were recorded at the frequency of $1000 \mathrm{~Hz}$ during the experiments by the haptic device and sensors.

Calculation of JND and Weber Fraction: It is a common way to fit a function to the experimental data to describe the dependence of an observers performance on some physical aspects of the stimulus in psychophysics. To determine a threshold, it is a common practice to fit a twoparameter function to the data and to compute the inverse of that function for the desired performance level [20], which 


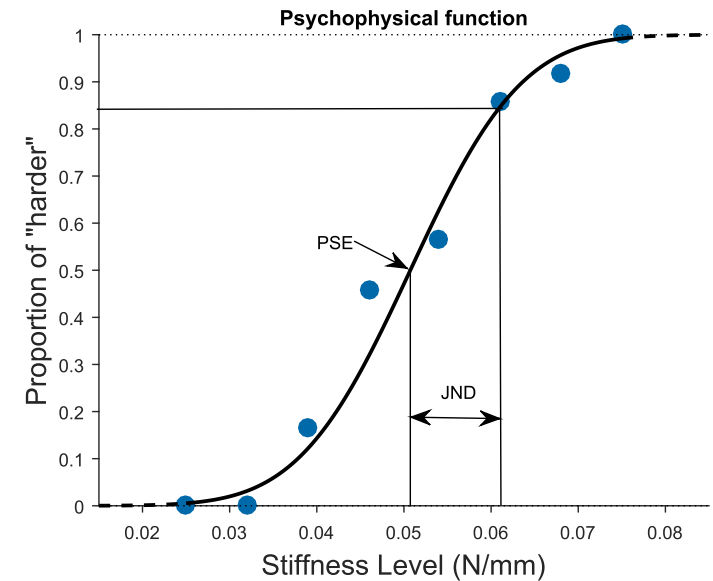

Fig. 3 A typical psychophysical function for experimental results. PSE is the point of subjective equality for reference and test stimuli. The JND is computed by taking the stimulus difference between PSE and proportion 0.84 according to the method of constant stimuli.

is typically a sigmoid function, such as the Weibull, logistic, cumulative Gaussian, or Gumbel distribution. The psychophysical functions fitted with a cumulative Gaussian function was used to obtain JNDs for the softness perception in this paper, whose goodness of fit and advantages had been verified by Wichmann [20] in two-alternative forced-choice situations. The function had been widely used to calculate the JNDs in the method of constant stimuli [13], [14], [21]. An example of this method for one of the reference stimuli (stiffness $=0.05 \mathrm{~N} / \mathrm{mm}$, damping $=0 \mathrm{Ns} / \mathrm{mm}$ ) is shown in Fig. 3, where the $\mathrm{X}$-axis represents the stimulus intensity and the Y-axis represents the subjects' performance, expressed as a proportion of "feeling harder than the reference" among the total number of choice per participant, and the data were fitted with the function:

$$
f(x)=\frac{1}{2}\left(1+\operatorname{erf}\left(\frac{x-\mu}{\sigma \sqrt{2}}\right)\right)
$$

where $\mu$ is a measure of the Point of Subjective Equality (PSE), which indicates the softness of the test stimulus that is perceived to be the same as the reference stimulus. And the width $\sigma$ of the function corresponds to the $84 \%$ level and is a measure for the discrimination threshold (JND). So the Weber Fraction could be calculated according to the Weber's law:

$$
\Delta I / I=K
$$

Here the $\Delta \mathrm{I}$ is the $\mathrm{JND}, I$ is the current stimulus intensity and $K$ is the Weber Fraction. The final Weber Fraction at a stimuli level is obtained by averaging all the subjects values.

Experiment 1: Measure of habitual exploring speed. The aim of experiment 1 was to investigate the habitual exploring speed of participants for experiment 2 . The brown speed guiding ball would not move and participants could move the blue ball unrestrained to exploring the softness at their habitual speeds. All the four stiffness levels $(0.025 \mathrm{~N} / \mathrm{mm}, 0.05 \mathrm{~N} / \mathrm{mm}, 0.075 \mathrm{~N} / \mathrm{mm}, 0.1 \mathrm{~N} / \mathrm{mm})$ without damping effect were tested and the JNDs were recorded for each participant and the order of tested stiffness level was also chosen pseudo-randomly.

Experiment 2: Stiffness rendering perception threshold. This experiment was designed to study whether the change of exploring speed would have some effect on the perceived JNDs, and select a proper guiding speed for the experiment 3 to eliminate the effect of speed changes on the damping force. So in experiment 2, the brown speed guiding ball would move up and down at a designed constant speed. Participants were asked to move the blue exploring ball following the guiding ball synchronously. According to the testing results of experiment 1 (habitual exploring speed $=79.8 \mathrm{~mm} / \mathrm{s}$ ), there are three speed levels (fast: $120 \mathrm{~mm} / \mathrm{s}$, normal: $80 \mathrm{~mm} / \mathrm{s}$, slow: $40 \mathrm{~mm} / \mathrm{s}$ ) for the participants to follow. The appearance of speed level for each participant also followed pseudo-random distribution. All the four stiffness levels $(0.025 \mathrm{~N} / \mathrm{mm}, 0.05 \mathrm{~N} / \mathrm{mm}, 0.075 \mathrm{~N} / \mathrm{mm}, 0.1 \mathrm{~N} / \mathrm{mm})$ without damping effect were tested here.

Experiment 3: Softness rendering perception with both stiffness and damping. Experiment 3 is aimed at investigating how the thresholds would change when perceiving softness generated by stiffness added with damping. There are four stiffness levels and four damping levels in total (stiffness: $0.025 \mathrm{~N} / \mathrm{mm}, 0.05 \mathrm{~N} / \mathrm{mm}, 0.075 \mathrm{~N} / \mathrm{mm}$, $0.1 \mathrm{~N} / \mathrm{mm}$, damping: $0.0025 \mathrm{Ns} / \mathrm{mm}, 0.005 \mathrm{Ns} / \mathrm{mm}$, $0.0075 \mathrm{Ns} / \mathrm{mm}, 0.01 \mathrm{Ns} / \mathrm{mm}$ ). They were combined and appeared as the reference stimuli randomly until the participant had perceived all the 16 kinds of conditions. The corresponding test stimulus had the same damping level as the reference while changing stiffness. In order to focus on the interaction effect between damping and stiffness and eliminate interference from speed factors, which may lead to different damping force and make the damping effect uncontrollable, the guiding speed was set up at the habitual speed $(80 \mathrm{~mm} / \mathrm{s})$ according to the results of experiment 2 . In this way, only exploring the softness at human habitual speed is taken into account in this paper.

\subsection{Results and Discussion}

Habitual Exploring speed: In experiment 1, subjects could explore the grey blocks freely. As shown in Fig. 4, most average freely exploring speeds of subjects are ranging from $60 \mathrm{~mm} / \mathrm{s}$ to $100 \mathrm{~mm} / \mathrm{s}$. The average free exploring speed of all subjects is $79.8 \mathrm{~mm} / \mathrm{s}$, which is regarded as the habitual speed of users when manipulating the device freely. So in experiment $2,80 \mathrm{~mm} / \mathrm{s}$ was chosen as the normal guiding speed and $40 \mathrm{~mm} / \mathrm{s}$ and $120 \mathrm{~mm} / \mathrm{s}$ were set as contrasts.

Effect of exploring speed on softness JNDs: Figure 5 shows the Weber Fraction of different exploring speed at the stiffness level of $0.1 \mathrm{~N} / \mathrm{mm}$, from which it can be inferred that low exploring speed $(40 \mathrm{~mm} / \mathrm{s})$ will slightly enhance the discrimination ability and the Weber Fractions of softness have no significant change under exploring speed of $80 \mathrm{~mm} / \mathrm{s}$ and $120 \mathrm{~mm} / \mathrm{s}$, which is consistent with previous researchresults conducted by Karadogan [22]. That indicates that the effect of exploring speed on the softness 


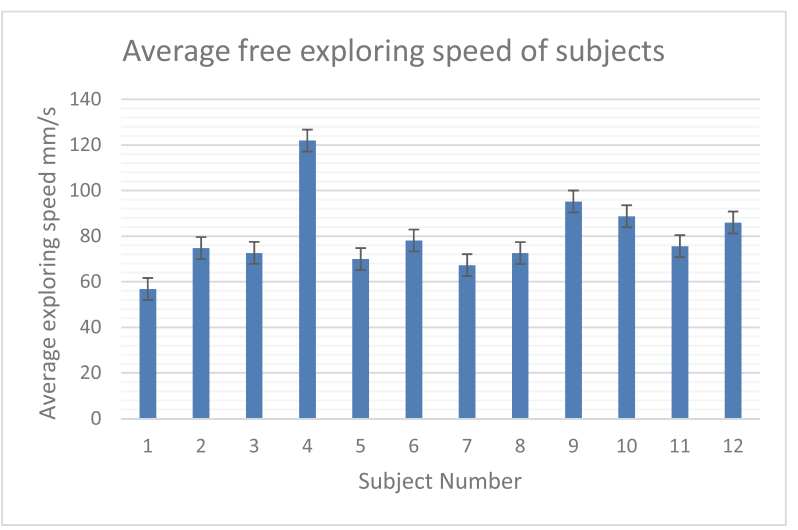

Fig. 4 Average free exploring speed of subjects. The error bars represent standard errors.

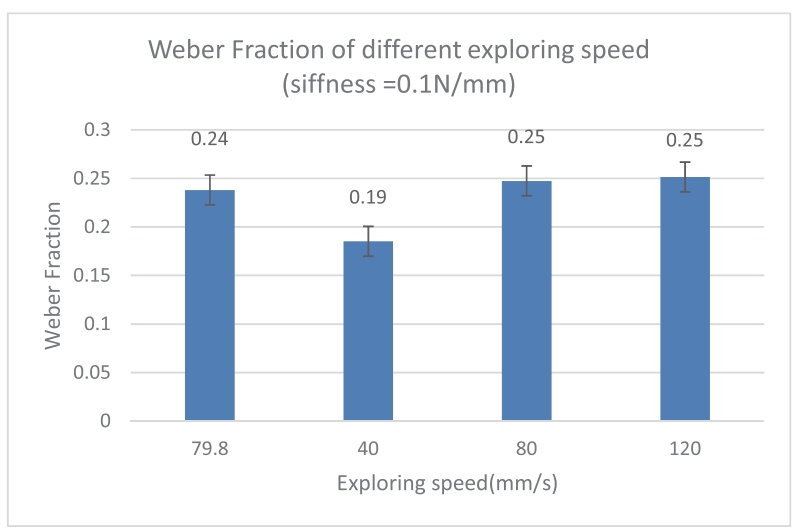

Fig. 5 Weber Fraction of perceived softness of different exploring speed. Perceived softness JNDs of stiffness level $0.1 \mathrm{~N} / \mathrm{mm}$ under different guiding exploring speed are shown here, taking Weber Fraction (0.24) of habitual speed $(79.8 \mathrm{~mm} / \mathrm{s})$ as a reference.

perception can be ignored when guiding users to explore the virtual environment close to habitual speed, which can be also confirmed by research of [23]. Therefore, in all the trails in experiment 3 , the guiding speed was set as $80 \mathrm{~mm} / \mathrm{s}$.

Softness rendering perception of combination of stiffness with damping: In experiment 3 , there are four stiffness levels and four damping levels in total. Before formal experiments that combining damping with stiffness, Weber Fractions of different stiffness levels at guiding speed of $80 \mathrm{~mm} / \mathrm{s}$ were tested firstly as contrasts for following analysis. As shown in Fig. 6, average Weber Fractions of four stiffness levels range from $0.22-0.25$, which is similar to Kuschels work (Weber Fraction=0.29) in 2010 [21], where they explored virtual cube with rigid probes. The difference is the range of stiffness levels due to performance limitations, which $(0.14-2.44 \mathrm{~N} / \mathrm{mm})$ were higher than work in this paper $(0-0.1 \mathrm{~N} / \mathrm{mm})$. But there is no evidence that the softness perception would deviate from Webers law when changing stiffness magnitudes in this range. In their work, they did not remove visual deformation information and the response time (2s) was much smaller than the time (30 s) in this paper, which would make the Weber Fractions different.

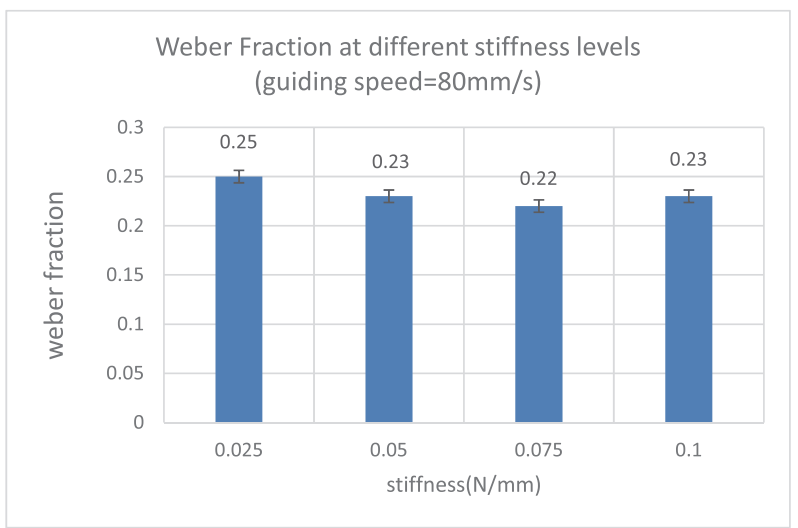

Fig. 6 Weber fraction at different stiffness levels. The guiding speed is $80 \mathrm{~mm} / \mathrm{s}$, which is nearly the same as participants' average habitual speed and the range of stiffness is $0-0.1 \mathrm{~N} / \mathrm{mm}$.

Table 1 Weber fractions of combination of different stiffness and damping

\begin{tabular}{|c|c|c|c|c|c|}
\hline $\begin{array}{l}\text { Damping } \\
\text { Stiffness } \\
(\mathrm{N} / \mathrm{N} \cdot \mathrm{s} / \mathrm{mm})\end{array}$ & 0 & 0.0025 & 0.005 & 0.0075 & 0.01 \\
\hline 0.025 & 0.25 & 0.35 & 0.35 & 0.39 & 0.36 \\
\hline 0.05 & 0.23 & $\mathbf{0 . 2 0}$ & $\mathbf{0 . 2 2}$ & 0.27 & 0.29 \\
\hline 0.075 & 0.22 & $\mathbf{0 . 1 5}$ & $\mathbf{0 . 1 6}$ & 0.22 & 0.22 \\
\hline 0.1 & 0.23 & $\mathbf{0 . 1 5}$ & $\mathbf{0 . 1 4}$ & $\mathbf{0 . 1 5}$ & $\mathbf{0 . 1 7}$ \\
\hline
\end{tabular}

Other Weber Fraction reported by related research is about $0.08-0.23$ [24]-[26], but most of there work were perceiving rigid or rubber objects by human finger directly, which were different conditions and got more information than the perception of softness rendering by rigid probe through haptic devices, so their values of Weber Fractions were usually smaller.

In the formal experiments, subjects were told to focus on feelings of softness if they had doubts about the current rendering effect. Average of Weber Fractions of combination of different stiffness and damping levels are shown in Table 1, and analysis results show that the stiffness level has significant effects on the Weber Fractions of perceived softness rendering injected with damping $([\mathrm{F}=89.42, \mathrm{p}<0.05])$, which is a contrary conclusion compared with perceived softness rendering without damping effect (as shown in the first column in Table 1). It is obvious that the contrary conclusion is caused by damping effect. A trend can be observed is that, for the same level of damping, increase of stiffness levels brings smaller Weber Fraction. For the same level of stiffness (except 0.025), Weber Fraction will decrease firstly and then increase when damping increase. Therefore, the ratio of damping to stiffness, which represents the ratio of damping force to stiffness force, was selected as an independent variable. And the relative threshold, which can be calculated as divide Weber Fraction of combination of different stiffness and damping by Weber Fraction of corresponding stiffness level without damping and reflected 


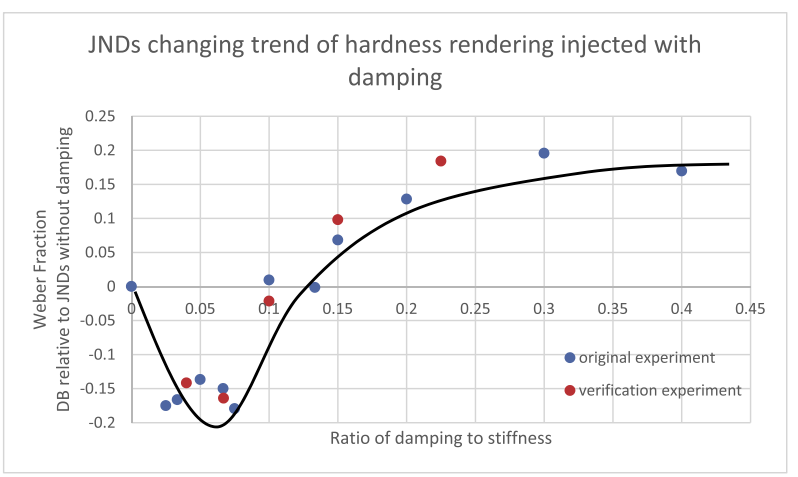

Fig. 7 Prediction curve for JNDs changing trend of softness rendering injected with damping. Blue dots are relative Weber fractions of different ratios of damping to stiffness in the experiment 3 , while red dots are relative Weber fractions in the verification experiment. Dark line is a possible prediction curve of changing trend of perceived softness threshold injected with damping effect.

the increase or decrease of the threshold, was selected as the dependent variable to find more clues.

It can be easily inferred that damping force plays a major role in softness rendering perception when the ratio is big enough while stiffness force plays major role in the softness rendering perception when the ratio is small. So it can be inferred that both the increase and decrease of Weber Fraction are bounded. The upper bound could be speculated because the feeling of viscosity caused by damping would dominate in the compliance perception when damping is much bigger than stiffness. So the measured Weber Fraction in this situation should be close to the value for viscosity effect alone, which is about 0.42 (bigger than softness). The Weber Fraction will decrease to the lower bound when the ratio is in a proper range and then increase when damping plays more important roles. A possible prediction curve for JNDs changing trend of softness rendering injected with damping is shown in Fig.7, from which the critical point of the ratio can be estimated as about $0.1-0.13$. There is an upper bound for the increasing of Weber Fraction when damping is much bigger than stiffness, and it's about $0.20 \mathrm{db}$ presumed by experimental results in this paper.

In order to verify the reliability and stability of the perceptual result, a verification experiment which covered different stiffness and damping levels was conducted in the same way compared with the experiment 3 in this paper. The stiffness levels selected in the verification experiment were $0.04 \mathrm{~N} / \mathrm{mm}, 0.06 \mathrm{~N} / \mathrm{mm}$ and $0.09 \mathrm{~N} / \mathrm{mm}$ while the damping levels selected in the verification experiment were $0.004 \mathrm{~N} \cdot \mathrm{s} / \mathrm{mm}, 0.006 \mathrm{~N} \cdot \mathrm{s} / \mathrm{mm}$ and $0.009 \mathrm{~N} \cdot \mathrm{s} / \mathrm{mm}$. Another 6 participants (4 male, 2 female) averaged 25.8 years old from Southeast University took part in the experiment. And the experimental conditions and the calculation of JNDs and Weber Fractions were the same as the original experiments. The perception results of the verification experiments were shown in Table 2 and Fig.7. It can be observed that the Weber Fractions have the same changing trend as the original experiment: decrease firstly and then increase when damp-
Table 2 Weber fractions of the verification experiment

\begin{tabular}{|c|c|c|c|c|}
\hline $\begin{array}{c}\text { Damping } \\
\text { Stiffness } \\
(\mathrm{N} / \mathrm{N} \cdot \mathrm{mm})\end{array}$ & 0 & 0.004 & 0.006 & 0.009 \\
\hline 0.04 & 0.22 & $\mathbf{0 . 2 0}$ & 0.31 & 0.34 \\
\hline 0.06 & 0.25 & $\mathbf{0 . 1 7}$ & $\mathbf{0 . 2 4}$ & 0.28 \\
\hline 0.09 & 0.24 & $\mathbf{0 . 1 7}$ & $\mathbf{0 . 1 7}$ & $\mathbf{0 . 2 3}$ \\
\hline
\end{tabular}

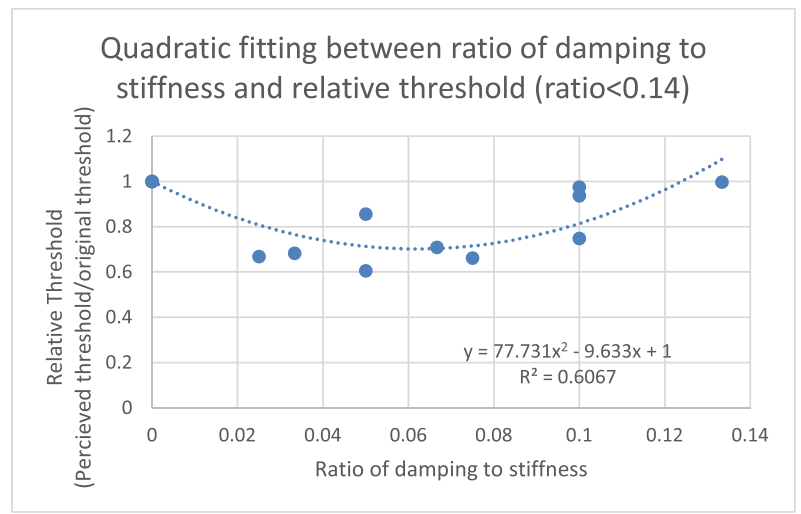

Fig.8 Quadratic fitting between ratio of damping to stiffness and relative threshold $($ ratio $<0.14)$

ing increase for the same stiffness level while increase of stiffness levels bring smaller Weber Fraction for the same damping level. And as shown in the Fig. 7, where new results were plot as red points together with original results, the relative Weber Fractions of results from the verification experiments could fit the prediction curve very well. These results verified the reliability of the experimental results in terms of compliance perception.

A quadratic fitting between ratio of damping to stiffness and relative threshold was conducted for the first part of the prediction curve (when the ratio is smaller than 0.14), as shown in Fig. 8, which indicated that only metric of ratio of damping to stiffness was not enough to express perceived softness very well $\left(R^{2}=0.6\right)$. More relevant physical metrics and the correlation analysis are needed to describe relation between objective physical parameters and subjective perceived results, which will be presented in part 3 .

\section{Useful Metrics, Parameters and Regression Analysis}

Haptic feeling of softness is a comprehensive perception result. Different quality metrics may represent different environmental factors. But how these quality metrics being combined to form the softness feeling is not clear, especially when taking damping information into account. Effect of experimental conditions such as object properties, interaction modes and human perception habits may be reflected when these metrics are expressed by basic experimental physical parameters such as the stiffness coefficient, damping, contact velocity and so on. So it is necessary to find out useful quality metrics in the perception of softness based on interaction data and analyze their expression of ba- 

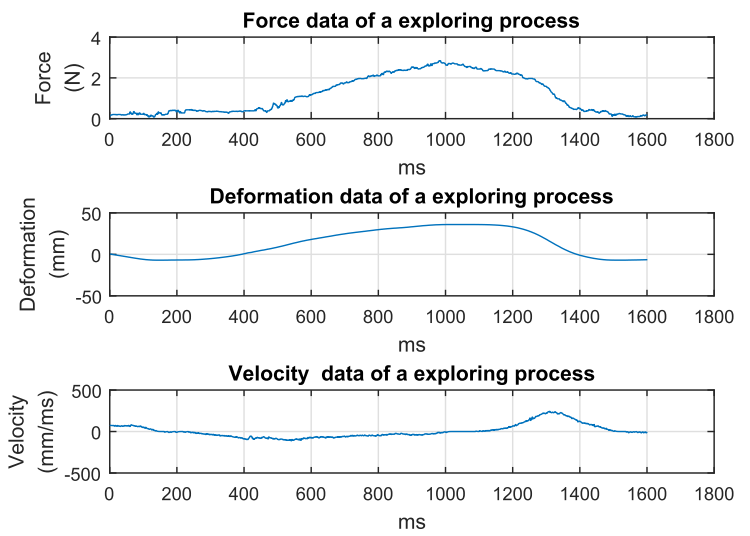

Fig. 9 Interaction data of an exploring process. Force, deformation, velocity data are recorded by sensors and haptic device synchronously. A complete exploring process consists of contact, pressing and release contact stages.
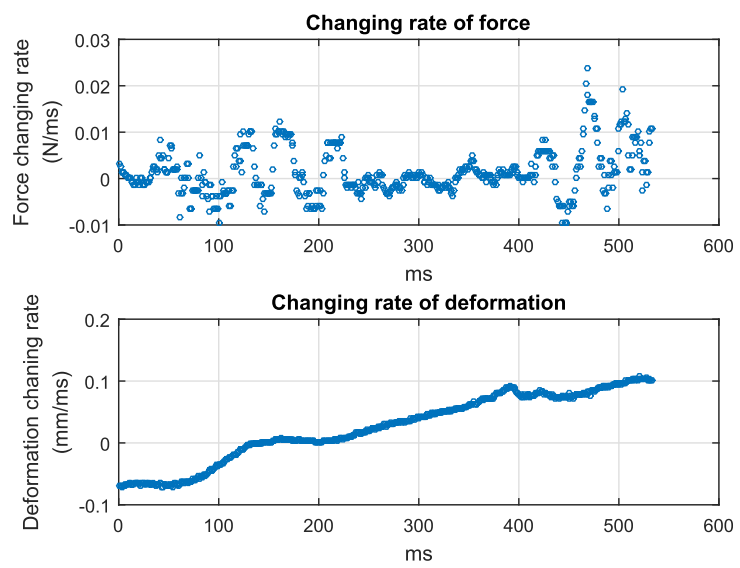

Fig. 10 Changing rate of force and deformation. Changing rate is calculated under the time window of $20 \mathrm{~ms}$ in this paper and the rate will change fast in a short time when the exploring state change, especially in initial contact state.

sic experimental physical parameters and figure out the correlations and physical meaning of them. And it would be significant to simulate the relations from physical parameters to subjective perception by mathematic models, which may provide useful help in design and improvement of haptic rendering devices.

\subsection{Force, Deformation, and Velocity Data}

Force, displacement, deformation, and velocity interaction data were recorded during each experimental trail. Interaction data of a typical exploring process are shown in Fig. 9. Based on previous researches [2], [15], [16], several popular metrics which may play important roles in the softness perception are shown in Fig. 10 and Fig. 11. It is worth mentioning that, here the metrics of Rate-hardness and extended Rate-hardness are not completely consistent with definitions. Rate-hardness $(\mathrm{RH})$ is defined as:

$$
R H=\frac{\text { Initial rate of change of force }(\mathrm{N} / \mathrm{s})}{\text { Initial penetration velocity }(\mathrm{ms})}
$$
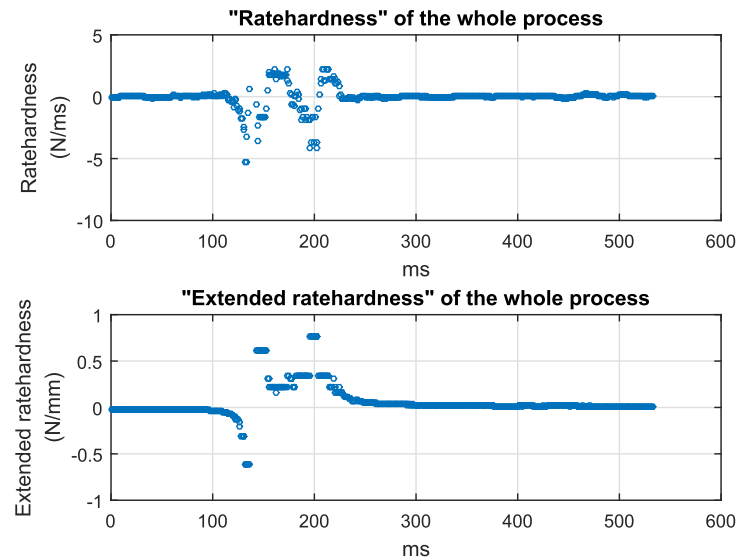

Fig. 11 Rate-hardness and extended rate hardness of a complete exploring process. Rate-hardness and extended rate-hardness are defined for initial contact stage, here the values are expanded to the whole process in order to find more possible physical metrics for perceived softness injected with damping.

While Extended Rate-hardness (ERH) is defined as:

$$
E R H=\frac{\text { Maximum rate of change of force }(\mathrm{N} / \mathrm{s})}{\text { Initial penetration velocity }(\mathrm{ms})}
$$

Here both of RH and ERH are single numerical values that reflect abrupt changing of contact stage. In Fig. 10, the concepts of RH and ERH are expanded to contact and pressing stages to show the changing trend more clearly. But in subsequent analysis work, values of RH and ERH are adopted as original definitions. In many situations, initial rate of force change equals the maximum rate of force change. But in some algorithms including a time difference between an initial contact and an abrupt rise of force response after the contact, they occur at different time just like the examples in this paper, as shown in Fig. 10. So RH and ERH are regarded as two different metrics to reflect more information during the exploring.

\subsection{Useful Quality Metrics}

In Hans [16] research, they examined the relevance of many physical variables obtained in experiments to the hardness perception. The metrics they selected were rendering method, measured stiffness, contact velocity, RH, ERH, peak force, and peak force rate. But they did not explain why the metrics are selected and what physical meaning they represent. As mentioned in Sect. 1, Bergmann Tiest and Kappers [13] quantitatively analyzed the contribution percent of kinaesthetic and cutaneous information to the hardness discrimination. They showed that about $90 \%$ of the information comes from surface deformation cues, and $10 \%$ from force/displacement cues. And in the situation of perceiving the softness rendering though rigid probe [23], most cutaneous clues come from the initial contact hit with the surface, and then decay rapidly, typically in $100 \mathrm{~ms}$. While 


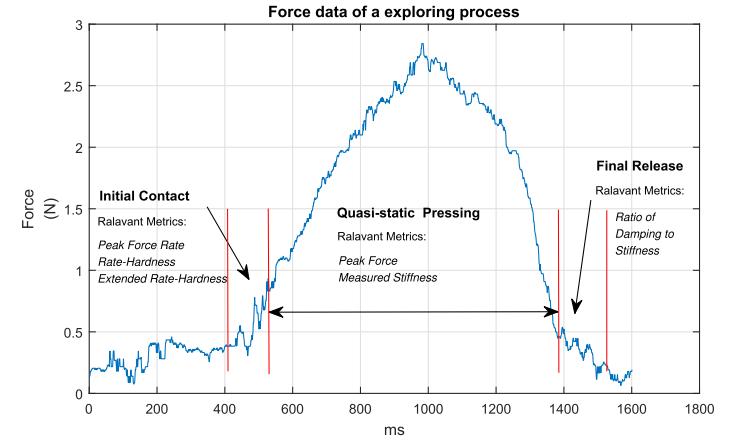

Fig. 12 Three sequential perceptual components of softness perception: initial dynamic contact, quasi-static pressing interaction, and the final dynamic release. And the distinct perceptual qualities of the three stages: the crispness of the initial contact, the hardness of the rigid surface, and the cleanness of the final release.

most kinaesthetic clues come from the pressing stage and are resulted from the apparent mechanical impedance of the object [27]. Some research [28] had also proposed that the basic perception of hardness has three sequential perceptual components: initial contact with the surface, quasi-static interaction with the hard surface, and the final dynamic release from the surface. They described the distinct perceptual qualities of the three stages as the crispness of the initial contact, the hardness of the rigid surface, and the cleanness of the final release and verified that the three perceptual qualities could describe the hardness feeling well, which are also corresponding to the stages that cutaneous and kinaesthetic clues come from. So in this paper, relevant metrics which may reflect these three perceptual qualities are selected based on the results of previous studies, as shown in Fig. 12.

\section{Crispness:}

Peak Force Rate: Peak Force Rate (PKR) is an important metric to reflect the crispness of the initial contact. Lamotte verified that change of the force applied to the skin by a tool was major tactile signals related to the objects compliance [23]. Friedman also proposed that peak and rate of force trace may provide useful cues used by subjects to rate softness [29]. Peak Force Rate was calculated by dividing the peak by the time taken to reach the peak. During initial contact stage, there would be a mutation of force within several milliseconds after contact and had a temporal profile that was sharpest for the hardest object and more rounded or gradual for softer objects, which could be reflected directly by the Peak Force Rate. It is possible that adding damping effect in the proper range would sharpen the force contour and make the softness easier to discriminate, which may be one of the reasons for the change of Weber Fraction.

Rate-Hardness and Extended Rate-Hardness: RateHardness and Extended Rate-Hardness have been proposed for a long time as important physical metrics to measure the softness perception. They have the same units but different physical meaning as stiffness. Different from PKR, they reflect the relation between force change and deformation change of the initial contact, which has high correlation with the crispness. Analysis from formula 1-3 showed that damping effect would also enhance the RH and ERH of the initial contact stage because the rate of deformation change would not be influenced by damping force. This probably could explain why damping effect is an additive to guarantee system stability, because of the conclusion that improvement of stability margins in the impedance rendered actually improved rate-hardness and perceived softness, which was obtained by Lawrence [15]. And when the damping effect is big enough, damping force would be a main factor instead of stiffness force not changing with deformation, which makes it difficult for participants to feel RH and ERH. This is also an important clues to explain the change of Weber Fraction in this paper.

\section{Rigidity:}

Measured Stiffness and Peak Force: In the quasistatic pressing process, force and deformation data change smoothly according to Hooke's law, as shown in Fig. 11. Measured Stiffness (MS) was a physical metric calculated by actual measured force and deformation data during pressing process. Peak Force (PK) is the maximum force value reached during the pressing process. Participants reached almost the same deformation because of the speed guiding ball in each trail. So PK is correlative to perceived softness. Damping effect in proper range may make the MS and PK bigger because it added a constant damping force would not change with deformation.

\section{Cleanness:}

Ratio of Damping to Stiffness: Cleanness is a perceptual quality of the final release stage. It can be inferred that it would be also influenced by RH and ERH. But it is more affected by the interaction effect of damping with stiffness. As mentioned above, damping force and stiffness force have opposite working directions that make the feeling of release stage more viscous. Ratio of Damping to Stiffness (DS) is metric that reflect which kind force is dominating in the perception. That is also an important reason for the discrimination ability change of the softness perception. Actually, DS works in all three stages but it is a dominating metric in the release stage.

\subsection{Correlation Analysis}

In order to show the change trend of Weber Fraction more clearly, relative threshold was also selected as the dependent variable to conduct the correlation analysis. Relative values represent increase or decrease degree of softness discrimination ability. The correlation matrix of physical metrics, perceived softness and perceived threshold are shown in Table 3 .

The table indicates that, for the human softness perception threshold (Weber Fraction) and relative threshold (increase or decrease degree of softness discrimination ability), physical metrics of Peak Force, Rate-Hardness, Extended Rate-Hardness and Ratio of Damping to Stiffness have high correlation (seen as values marked by bold font in the last two lines in the table) and they show nearly the same mag- 
Table 3 Correlation matrix of physical metrics, perceived softness and threshold

\begin{tabular}{|c|c|c|c|c|c|c|c|c|c|c|c|}
\hline & Physical metrics & 1 & 2 & 3 & 4 & 5 & 6 & 7 & 8 & 9 & 10 \\
\hline & (1)Stiffness & 1.00 & & & & & & & & & \\
\hline & (2)Damping & 0.00 & 1.00 & & & & & & & & \\
\hline \multirow{3}{*}{ Crispness metrics } & (3)Peak Force Rate & 0.10 & -0.46 & 1.00 & & & & & & & \\
\hline & (4)Rate-Hardness & -0.15 & -0.01 & 0.02 & 1.00 & & & & & & \\
\hline & (5)Extended Rate-Hardness & 0.03 & 0.52 & 0.02 & 0.22 & 1.00 & & & & & \\
\hline \multirow{2}{*}{ Rigidity Metrics } & (6)Measured Stiffness & 0.21 & 0.19 & 0.38 & -0.50 & 0.41 & 1.00 & & & & \\
\hline & (7)Peak Force & 0.11 & -0.19 & $\overline{0.40}$ & -0.62 & 0.13 & $\overline{0.73}$ & 1.00 & & & \\
\hline Cleanness Metrics & (8)Ratio of Damping to Stiffness & -0.11 & 0.72 & -0.43 & 0.48 & 0.65 & -0.12 & -0.40 & 1.00 & & \\
\hline \multirow{2}{*}{ Perceived Results } & (9)Softness Threshold (JND) & -0.14 & 0.19 & -0.09 & 0.73 & 0.47 & -0.28 & -0.46 & 0.72 & 1.00 & \\
\hline & (10)Relative Threshold & -0.07 & -0.20 & -0.06 & 0.70 & 0.49 & -0.22 & -0.43 & 0.72 & 1.00 & 1.00 \\
\hline
\end{tabular}

nitudes changing law, that means that softness threshold and relative threshold can be regarded as one kind of perceived result, which can also be proved by the correlation coefficient (1.0) between them. The other variables (Peak Force Rate and Measured Stiffness) show very low or little correlation with perceived softness or threshold (all less than 0.38, nearly the same as Hans [16] research). Especially, Measured Stiffness has low correlation $(-0.28$ and -0.22$)$ with softness perceived results, which also proved that kinaesthetic occupied a small proportion in softness rendering perception. Peak Force Rate has low correlation with perceived result (-0.09 and -0.06), which indicates that adding damping effect only sharpened the force contour but not changed the rate of force changing. DS almost has high correlation with all metrics of crispness and cleanness, that means that damping effect mainly works on initial contact and final release process and have little effect on quasi-static pressing process.

\subsection{Regression Analysis and Roles of Physical Parameters}

In order to verify the correlation analysis results in Table 3 further and build a perception model to describe the relations, relative perceived softness and relative threshold was selected as dependent variables respectively and corresponding metrics having high correlation coefficient were selected as independent variables to conduct regression analysis based on correlation matrix table above. It is worth noting that, RH and ERH have high correlation with DS, 0.48 and 0.65 respectively, RH also has high correlation with peak force $(-0.62)$, as seen in Table 3 , so the interaction effect between metrics can not be ignored when expressing perceived softness and threshold with these metrics. What's more, there are presumably nonlinear relationships between the physical metrics and perceived softness, so full quadratic of the variables are considered in the stepwise regression. If physical metrics of DS, Peak Force, $\mathrm{RH}$, ERH, Relative Threshold are expressed as DS, PF, RH, ERH, and $W F_{r}$ respectively, then regression analysis results for relative threshold are shown in Table 4 and Table 5. DS, PF, RH, and ERH are all significant metrics for relative threshold and full quadratic (14 variables in total) of the five metrics are taken into account. Variables whose p-values smaller than 0.05 are selected to express the perception results.
Table 4 Coefficients and p-values of significant metrics for relative threshold

\begin{tabular}{|c|c|c|c|}
\hline Variables & Coefficient & $\mathrm{t}$-stat & $\mathrm{p}$-value \\
\hline PF & -0.0272 & -0.4347 & 0.6709 \\
\hline RH & 0.2557 & 0.3687 & 0.7183 \\
\hline ERH & 0.5751 & 3.5736 & 0.0031 \\
\hline DS & 1.2615 & 1.3868 & 0.1888 \\
\hline$P F^{2}$ & -0.0047 & -0.3380 & 0.7048 \\
\hline$R H^{2}$ & 2.1033 & 6.1422 & $<0.0001$ \\
\hline$E R H^{2}$ & -0.0601 & -2.3498 & 0.0340 \\
\hline$D S^{2}$ & 12.0414 & 4.8925 & 0.0002 \\
\hline$P F \times R H$ & -0.1227 & -0.4720 & 0.6448 \\
\hline$P F \times E R H$ & -0.0039 & -0.1437 & 0.8880 \\
\hline$P F \times D S$ & 0.4347 & 0.7663 & 0.4572 \\
\hline$R H \times E R H$ & -0.7727 & -4.7185 & 0.0003 \\
\hline$R H \times D S$ & 2.8580 & 1.5426 & 0.1469 \\
\hline$E R H \times D S$ & -0.6904 & -0.1395 & 0.8912 \\
\hline
\end{tabular}

Table 5 Results of stepwise regression for softness perception (confidence 0.05)

\begin{tabular}{|c|c|c|c|c|c|}
\hline Intercept & 0.1727 & $\mathrm{R}^{2}$ & 0.8709 & $\mathrm{~F}$ & 18.8955 \\
\hline RMSE & 0.1244 & Adj $^{2}$ & 0.8248 & $\mathrm{p}$ & $8.8723 \mathrm{e}-06$ \\
\hline
\end{tabular}

As shown in Table 5, the $\mathrm{R}^{2}$ and adjusted $\mathrm{R}^{2}$ are 0.8709 and $0.8248, \mathrm{p}$ is $8.8723 \mathrm{e}-06(<0.05)$, that means that the independent variables can explain the dependent variables very well and the equation are effective, which also confirmed that correlation analysis results in Table 3. So the regression equation between $W F_{r}$ and $\mathrm{PF}, \mathrm{RH}, \mathrm{ERH}$ and DS can be expressed as:

$$
\begin{gathered}
W F_{r}=0.5751 E R H+2.1033 R H^{2} \\
-0.0601 E R H^{2}+12.0414 D S^{2} \\
-0.7727 R H \times E R H+0.1727
\end{gathered}
$$

It is worth noting that, Peak Force does not play an impor- 
tant role in the softness perception with damping result according to the regression analysis results. Peak force and peak force rate were regarded as important measures for the softness perception by some researchers [23], but some work [16], [29] got the conclusion that they were irrelevant to softness perception, which is in accordance with the conclusion of this paper.

$\mathrm{RH}, \mathrm{ERH}, \mathrm{DS}$ and their interaction are significant in the perception of softness. They are related with change of force and deformation and reflect the crispness of initial contact and cleanness during the exploring. Expressions of force $(f)$, RH and ERH are shown in formula 1-3. However, as described by Lawrence [15], the initial rate-of-change of force due to penetration with initial velocity is infinite and $f$ is discontinuous on the surface boundary when the force is zero before contact. In practice, this surface (control law) is not implementable since the derivative cannot be exactly computed. So in this paper, an example of low-pass filtered version of the control law was taken into account to gain some insight into the roles of physical parameters as Lawrence [15] did:

$$
\begin{aligned}
& f=\frac{(B s+K)}{(s / a+1)} \delta x(s) \\
& \dot{f}(t)=-a f(t)+a B \dot{\delta} x(t)+a K(\delta x(t))
\end{aligned}
$$

Here $a$ is the bandwidth with which forces can be supplied by the haptic devices, and the deformation $\delta x(t)=x(t)-x_{0}$. So with an initial penetration velocity $v_{0}$, the RH and ERH can be expressed as:

$$
\begin{aligned}
& R H=\frac{\dot{f}(0)}{\dot{x}(0)}=\frac{a B \dot{\delta} x(0)}{\dot{x}(0)}=a B \\
& E R H=\frac{\max (\dot{f}(t))}{\dot{x}(0)}=\frac{\max (\dot{f}(t))}{v_{0}}
\end{aligned}
$$

Lawrence had analyzed that when $K / B=a, f(s)=K \delta x(s)$ and the perceptual softness is equal to $K$. It means that the condition for significant damping effect is $B / K \geq 1 / a$. Combined with the conclusions and experiments of this paper, it can be inferred that the approximate value of 1/a in this paper is about $0.1-0.13$. And the probable relation between Weber Fraction and physical parameters can be expressed as:

$$
\begin{gathered}
W F_{r}=0.5751 \frac{\max (\dot{f}(t))}{v_{0}}+2.1033(a B)^{2} \\
-0.0601\left(\frac{\max (\dot{f}(t))}{v_{0}}\right)^{2}+12.0414\left(\frac{B}{K}\right)^{2} \\
-0.7727 a B \frac{\max (\dot{f}(t))}{v_{0}}+0.1727
\end{gathered}
$$

Formula 8 indicates that the discrimination ability for softness (Weber Fraction) is not only correlative with physical parameters for attributes of the object $(B, K)$, but also correlative with parameters of exploring condition (initial speed $v_{0}$, meaning habitual exploring speed in this paper) and haptic rendering devices (bandwidth $a$ ). It's worth noting that, the appearance of $\max (\dot{f}(t))$ will also be influenced by human exploring habit because it has high correlation with human active velocity compensation when the speed decrease after the initial contact. So discrimination ability for softness is a combination effect of attributes of the object, interaction environment and human perception habit. Specifically, the values of $B / K$ and $1 / a$ determine whether damping effect is significant in softness perception, which influences the cleanness. $v_{0}$ and $\max (\dot{f}(t))$ reflect the contour of force change is sharp or flat, which affects crispness. All of these physical parameters should be reckoned in haptic rendering, haptic perception and data analysis for softness.

\section{Conclusions}

Based on the results of experiments, conclusions can be speculated that the user habitual exploring speed for softness through Geomagic Touch is about $80 \mathrm{~mm} / \mathrm{s}$ in the experimental condition of this paper and exploring speed has slightly effect on the subjective perception of softness. Significantly reducing the exploring speed can increase the sensitivity of softness perception. Perceived softness JNDs would decrease firstly and then increase when the ratio of damping to stiffness increase and the boundary value for Weber Fraction increasing or decreasing of DS is about 0.10.13 . The increase and decrease of the softness perception ability are bounded. The upper bound of Weber Fraction is about 0.42 and the lower bound is about 0.15 for softness rendering perception. Further analysis indicates that the the boundary value for Weber Fraction increasing or decreasing of DS is influenced by force bandwidth $a$, which is decided by the haptic rendering device and algorithm.

A lot of useful quality metrics are analyzed to see whether they are important cues in the softness perception. For the human softness perception threshold (Weber Fraction), physical metrics of Rate-Hardness, Extended RateHardness and Ratio of Damping to Stiffness have high correlations. Measured stiffness, Peak Force has low correlation with softness perceived results, which also proved that kinaesthetic information occupied a small proportion in softness rendering perception. Peak Force Rate has low correlation with perceived result, that indicates that adding damping effect only sharpened the force contour but didn't change the Peak Force Rate. DS almost has high correlation with all metrics of crispness and cleanness, which means that damping effect mainly works on initial contact and final release process and has little effect on quasi-static pressing process. Damping effect in proper range would make the contour of force change sharper, which improves crispness (expressed by metrics of RH and ERH) and cleanness (expressed by DS) and make the softness easier to discriminate and increased system stability. When the damping level is big enough to make the damping force play a major role in the perception, perceived feeling tends to be viscosity instead of softness, thereby affecting the softness rendering perception threshold.

Further analysis results show that change of these rele- 
vant quality metrics is caused by basic physical parameters of different experimental conditions. The regression model between physical parameters and perceived JNDs indicates that discrimination ability for softness is a combination effect of the attributes of the object (parameters of $K, B$ ), the interaction environment (parameters of $a$ ) and the human perception habit (parameters of $v_{0}, \max (\dot{f}(t))$. Basic physical experimental parameters lead to the change of relevant quality metrics, then bring different perceptual feeling of interaction process (crispness and cleanness) and finally result in change of discrimination ability (JNDs). The relation between occurrence of $\max (\dot{f}(t))$ and experimental environment or perceptual habit is an issue worth studying in the future. What's more, the exploring speed is set as human habitual exploring speed in this paper. How the constant coefficients of these parameters will change when exploring softness at other speed is another significant issue to research. Findings in this paper may provide a better understanding of softness perception and useful guidance in improvement of haptic devices.

Certainly, though some verification experiments and the theory analysis have been conducted to verify how the results are sound in terms of perception in this paper, some more tests of specific applications and examples are necessary to show the actual effect in compliance rendering and perception, which is the focus of our current research.

\section{Acknowledgments}

This research was supported by National Natural Science Foundation of China under grants 61473088 , State Key Laboratory for Bioelectronics and Jiangsu Key Laboratory for Remote Measurement and Control.

\section{References}

[1] W.M. Bergmann Tiest and A.M. Kappers, "Cues for haptic perception of compliance," IEEE Transactions on Haptics, vol.2, no.4, pp.189-199, 2009.

[2] W.M. Bergmann Tiest, "Tactual perception of material properties," Vision research, vol.50, no.24, pp.2775-2782, 2010.

[3] P.F. Hokayem and M.W. Spong, "Bilateral teleoperation: An historical survey," Automatica, vol.42, no.12, pp.2035-2057, 2006.

[4] E. Nuño, L. Basañez, and R. Ortega, "Passivity-based control for bilateral teleoperation: A tutorial," Automatica, vol.47, no.3, pp.485-495, 2011.

[5] D. Sun, F. Naghdy, and H. Du, "Application of wave-variable control to bilateral teleoperation systems: A survey," Annual Reviews in Control, vol.38, no.1, pp.12-31, 2014.

[6] E. Nuño, L. Basañez, R. Ortega, and M.W. Spong, "Position tracking for non-linear teleoperators with variable time delay," The International Journal of Robotics Research, vol.28, no.7, pp.895-910, 2009.

[7] R. Harper and S. Stevens, "Subjective hardness of compliant materials," Quarterly Journal of Experimental Psychology, vol.16, no.3, pp.204-215, 1964.

[8] H.Z. Tan, X.D. Pang, N.I. Durlach, et al., "Manual resolution of length, force, and compliance," Advances in Robotics, vol.42, pp.13-18, 1992.

[9] H.Z. Tan, N.I. Durlach, Y. Shao, and M. Wei, "Manual resolution of compliance when work and force cues are minimized," ASME Dyn.
Syst. Contr. Div. Publ. DSC, vol.49, pp.99-104, 1993.

[10] U. Koçak, K.L. Palmerius, C. Forsell, A. Ynnerman, and M. Cooper, "Analysis of the jnd of stiffness in three modes of comparison," International Workshop on Haptic and Audio Interaction Design, pp.22-31, Springer, 2011.

[11] F.K. Freyberger and B. Färber, "Compliance discrimination of deformable objects by squeezing with one and two fingers," Proc. EuroHaptics, pp.271-276, 2006.

[12] M.A. Srinivasan and R.H. LaMotte, "Tactual discrimination of softness," Journal of Neurophysiology, vol.73, no.1, pp.88-101, 1995.

[13] W.M. Bergmann Tiest and A.M. Kappers, "Kinaesthetic and cutaneous contributions to the perception of compressibility," International Conference on Human Haptic Sensing and Touch Enabled Computer Applications, pp.255-264, 2008.

[14] F.E. van Beek, D.J. Heck, H. Nijmeijer, W.M. Bergmann Tiest, and A.M. Kappers, "The effect of global and local damping on the perception of hardness," IEEE Transactions on Haptics, no.3, pp.409420, 2016

[15] D.A. Lawrence, L.Y. Pao, A.M. Dougherty, M.A. Salada, and Y. Pavlou, "Rate-hardness: A new performance metric for haptic interfaces," IEEE Trans. Robot. Autom., vol.16, no.4, pp.357-371, 2000.

[16] G. Han and S. Choi, "Extended rate-hardness: A measure for perceived hardness," International Conference on Human Haptic Sensing and Touch Enabled Computer Applications, pp.117-124, 2010.

[17] S.C. Hauser and G.J. Gerling, "Force-rate cues reduce object deformation necessary to discriminate compliances harder than the skin," IEEE Transactions on Haptics, vol.11, no.2, pp.232-240, 2018.

[18] K. Higashi, S. Okamoto, Y. Yamada, H. Nagano, and M. Konyo, "Hardness perception through tapping: peak and impulse of the reaction force reflect the subjective hardness," International Conference on Human Haptic Sensing and Touch Enabled Computer Applications, pp.366-375, 2018.

[19] M.O. Ernst and M.S. Banks, "Humans integrate visual and haptic information in a statistically optimal fashion," Nature, vol.415, no.6870, p.429, 2002.

[20] F.A. Wichmann and N.J. Hill, "The psychometric function: I. fitting, sampling, and goodness of fit," Perception \&amp; psychophysics, vol.63, no.8, pp.1293-1313, 2001.

[21] M. Kuschel, M. Di Luca, M. Buss, and R.L. Klatzky, "Combination and integration in the perception of visual-haptic compliance information," IEEE Transactions on Haptics, vol.3, no.4, pp.234-244, 2010.

[22] E. Karadogan, R.L. Williams, J.N. Howell, R.R. Conatser, Jr, et al., "A stiffness discrimination experiment including analysis of palpation forces and velocities," Simulation in Healthcare, vol.5, no.5, pp.279-288, 2010.

[23] R.H. LaMotte, "Softness discrimination with a tool," Journal of Neurophysiology, vol.83, no.4, pp.1777-1786, 2000.

[24] H.Z. Tan, N.I. Durlach, G.L. Beauregard, and M.A. Srinivasan, "Manual discrimination of compliance using active pinch grasp: The roles of force and work cues," Perception \& psychophysics, vol.57, no.4, pp.495-510, 1995.

[25] L.A. Jones and I.W. Hunter, "A perceptual analysis of stiffness," Experimental Brain Research, vol.79, no.1, pp.150-156, 1990.

[26] F.K. Freyberger and B. Farber, "Psychophysics and perceiving granularity," 2006 14th Symposium on Haptic Interfaces for Virtual Environment and Teleoperator Systems, pp.387-393, IEEE, 2006.

[27] G. Han, S. Jeon, and S. Choi, "Improving perceived hardness of haptic rendering via stiffness shifting: an initial study," Proc. 16th ACM Symposium on Virtual Reality Software and Technology, pp.87-90, ACM, 2009.

[28] L.B. Rosenberg and B.D. Adelstein, "Perceptual decomposition of virtual haptic surfaces," Virtual Reality, 1993. Proceedings., IEEE 1993 Symposium on Research Frontiers in, pp.46-53, IEEE, 1993.

[29] R.M. Friedman, K.D. Hester, B.G. Green, and R.H. LaMotte, "Magnitude estimation of softness," Experimental brain research, vol.191, no.2, pp.133-142, 2008. 

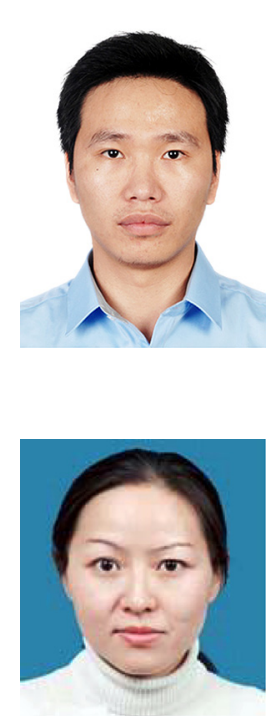

Zhiyu Shao received the bachelors degree in instrument science and engineering from Southeast University, Nanjing, China, in 2014, where he is currently working toward the $\mathrm{PhD}$ degree in the State Key Laboratory for Bioelectronics, School of Instrument Science and Engineering. His research interests include haptic rendering, human-computer interaction, machine learning system and neural network.

Juan Wu received the BS, MS, and $\mathrm{PhD}$ degrees from the School of Instrument Science and Engineering of Southeast University, Nanjing, China, in 1999, 2002, and 2005, respectively. She is currently a professor in the School of Instrument Science and Engineering, Southeast University. Her research interests include robotic sensors and control, haptic rendering and virtual reality. She is a member of the IEEE and a member of the IEEE Robotics and Automation Society (RAS).

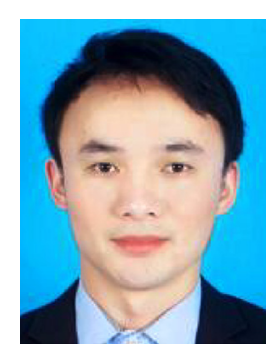

Qiangqiang Ouyang received the BS and MS degrees from the school of electrical and information engineering, Anhui university of technology, Maanshan, China, in 2012 and 2016, respectively. He is currently working toward the PHDs degree in the School of Instrument Science and Engineering, Southeast University. His research interests include Modeling and application of tactile illusion. 